\title{
Antioxidants in Oral Health and Diseases: Future Prospects
}

\author{
Dr. Nimmi Singh, Dr. Rajarshi Guha Niyogi, Dr Deepak Mishra, \\ Dr. Mamta Sharma, Dr. Devika Singh \\ Department of Oral Medicine \& Radiology B.I.D.S.H, Patna \\ Department of Oral Medicine \& Radiology B.I.D.S.H, Patna \\ ,RIO,IGIMS,Patna,Bihar \\ Indira Gandhi Government Dental College \& Hospital, Jammu \\ Post graduate. Department of Oral Medicine \& Radiology, S.P.P.I.D.M.S, Lucknow
}

\begin{abstract}
Free radicals have been implicated in the etiology of large number of major diseases. They can adversely alter many crucial biological molecules leading to loss of form and function. Such undesirable changes in the body can lead to diseased conditions. Antioxidants are compounds which destroy the free radicals in the body, thereby preventing against harmful oxidation- reduction reactions. The best sources are fruits and vegetables which provide a variety of antioxidants like Vit. A, C, E \& carotenoids. Currently available data are compatible with the notion that these vitamins act as chemopreventives against some important cancers, e.g. carotenoids for lung cancer, ascorbic acid for salivary gland cancer, tocopherols for head and neck cancers etc. Thus, a greater consumption of fruits and vegetables should be encouraged as they are the natural sources of these chemopreventive antioxidants. This paper will briefly review the basics of antioxidants and various scientific studies that bear out the promise of antioxidants for oral care \& diseases.
\end{abstract}

Key words: Antioxidants, chemopreventive, free radicals, carotenoids.

\section{Introduction}

Oxygen is an element indispensable for life. When cells use oxygen to generate energy, free radicals are created as a consequence of ATP (adenosine triphosphate) production by the mitochondria. These byproducts are generally reactive oxygen species (ROS) as well as reactive nitrogen species (RNS) At high concentrations, they generate oxidative stress, a deleterious process that can damage all cell structures ${ }^{1}$. Oxidative stress plays a major part in the development of chronic and degenerative ailments such as cancer, arthritis, aging, autoimmune disorders, cardiovascular and neurodegenerative disease The human body has several mechanisms to counteract oxidative stress by producing antioxidants, which are either naturally produced in situ, or externally supplied through foods and/or supplements. Endogenous and exogenous antioxidants act as "free radical scavengers" by preventing and repairing damages caused by ROS and RNS, and therefore can enhance the immune defense and lower the risk of cancer and degenerative diseases ${ }^{2}$.

\section{Mechanism of action of free radicals}

Free radicals are chemically active atoms that have a charge due to an excess or deficient number of electrons. Free radicals containing oxygen, known as reactive oxygen species (ROS), are the most biologically significant free radicals. ${ }^{3}$ In the recent years the term "Reactive Oxygen Species" or "Reactive Oxygen Intermediates" is a collective term which has been adopted to include molecules like Hydroxyl radical $(. \mathrm{OH})$,Superoxide anion $(\mathrm{O} 2)$, Hydrogen peroxide $(\mathrm{H} 2 \mathrm{O} 2)$, Hypochlorous acid (HCIO) ${ }^{4}$

While most reactive oxygen species have extremely short half life, they can cause substantial tissue damage by initiating free radical chain reaction. Reactive oxygen species can cause tissue damage by a variety of different mechanism which include;

- DNA damage

- Lipid peroxidation (through activation of cyclogenase and lipo oxygenase pathway)

- Protein damage including gingival hyaluronic acid and proteoglycans

- Oxidation of important enzymes eg Antiprotease such as ; 1 antitrypsin

- Stimulation of pro inflammatory cytokine release by monocytes and macrophages by depleting intracellular thiol compounds and activating nuclear factor 


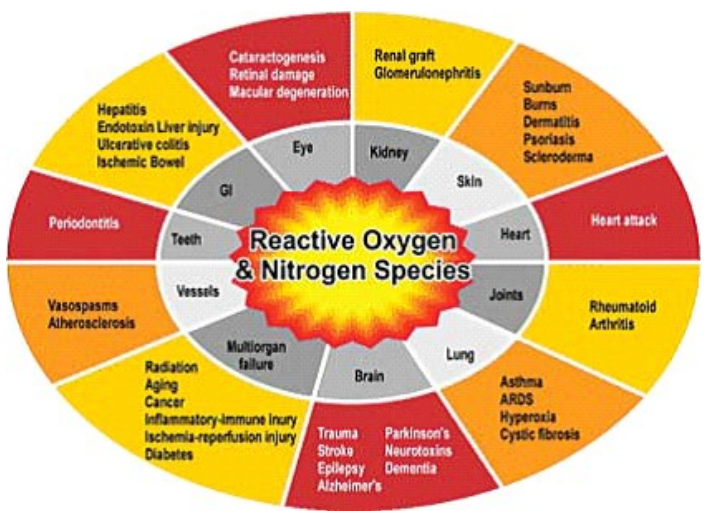

Diseases caused by free radicals

\section{Classifications}

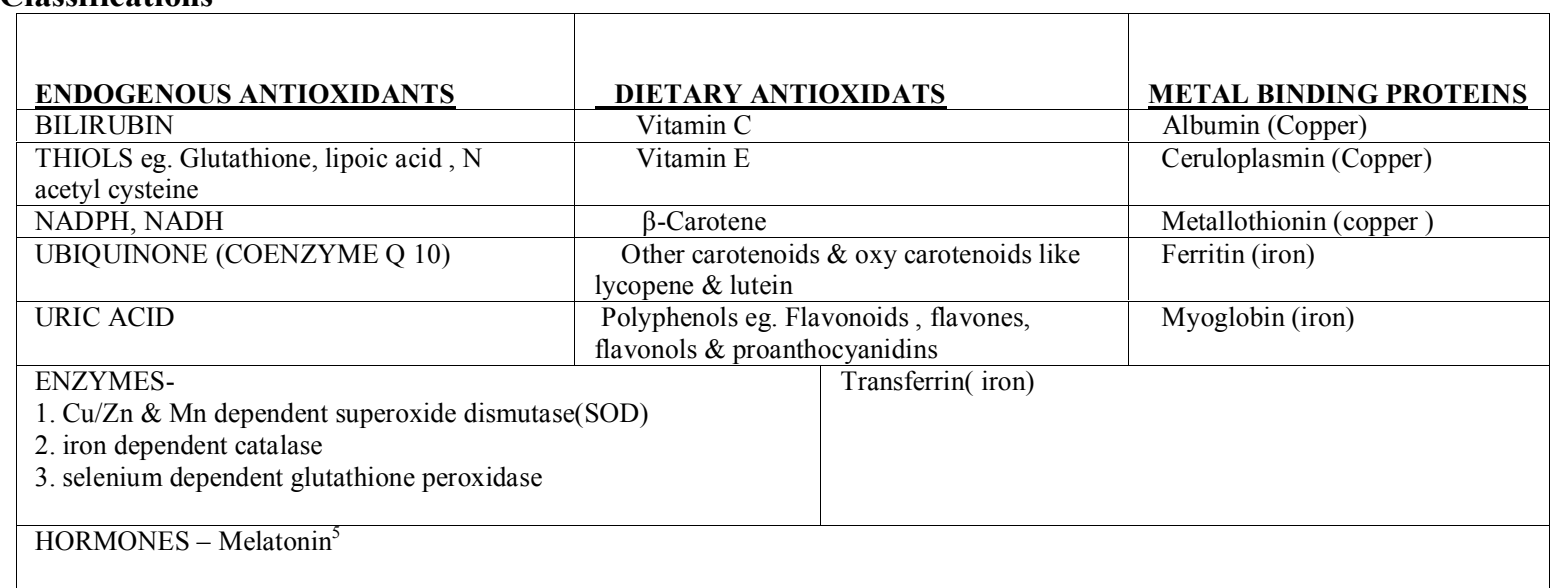

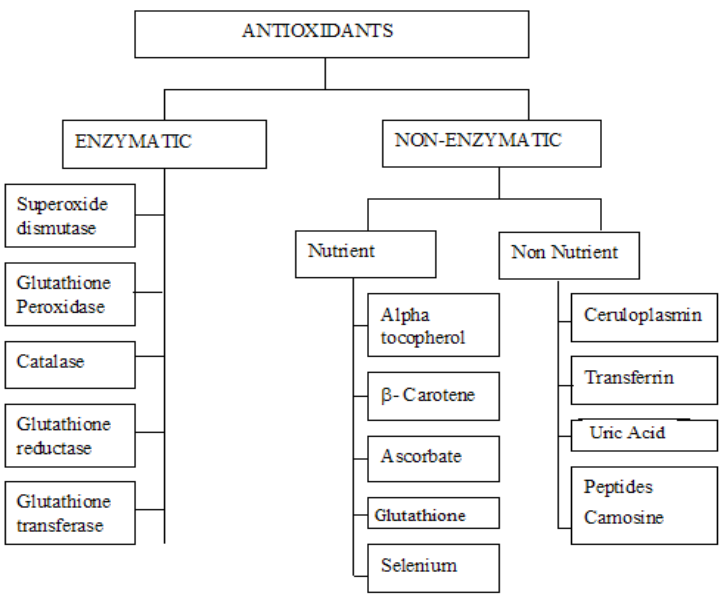

Iain L, C. Chapple et al

\section{Types of antioxidants}

Antioxidants from our diet play an important role in helping endogenous antioxidants for the neutralization of oxidative stress.

Vitamin C Vitamin C also known as ascorbic acid, is a water-soluble vitamin. It is essential for collagen, carnitine and neurotransmitters biosynthesis. Health benefits of vitamin $\mathrm{C}$ are antioxidant, anti-atherogenic, anticarcinogenic, immunomodulator. ${ }^{7}$ Vitamin $\mathrm{C}$ works synergistically with vitamin $\mathrm{E}$ to quench free radicals and also regenerates the reduced form of vitamin E. Natural sources of vitamin C are acid fruits, green vegetables, tomatoes ${ }^{3}$.

Vitamin E -Vitamin $\mathrm{E}$ is a fat-soluble vitamin with high antioxidant potency. Because it is fat-soluble, $\alpha$ tocopherol safeguards cell membranes from damage by free radicals. Its antioxidant function mainly resides in the protection against lipid peroxidation. The dietary sources of vitamin E are vegetable oils, wheat germ oil, whole grains, nuts, cereals, fruits, eggs, poultry meat ${ }^{8}$. Vitamin $\mathrm{E}$ has been proposed for the prevention against 
colon, prostate and breast cancers, some cardiovascular diseases, ischemia, cataract, arthritis and certain neurological disorders.'

Beta-carotene- Beta-carotene is a fat soluble member of the carotenoids which are considered provitamins because they can be converted to active vitamin A. Beta-carotene is converted to retinol, which is essential for vision. It is a strong antioxidant and is the best quencher of singlet oxygen. Beta-carotene is present in many fruits, grains, oil and vegetables (carrots, green plants, squash, spinach) ${ }^{5}$

Selenium- Selenium is a trace element. It forms the active site of several antioxidant enzymes including glutathione peroxidase. Similar to selenium, the minerals manganese and zinc are trace elements that form an essential part of various antioxidant enzymes. ${ }^{3}$ Se is a trace mineral found in soil, water,

vegetables (garlic, onion, grains, nuts, soybean), sea food,meat, liver, yeast. ${ }^{10}$ At low dose, health benefits of Se are antioxidant, anti-carcinogenic and immunomodulators. ${ }^{11}$

Lycopene- Lycopene has been hypothesized to prevent carcinogenesis and atherogenesis by protecting critical cellular biomolecules, including lipids, lipoproteins, proteins, and DNA. Lycopene, when given in the dosage of $4.8 \mathrm{mg}$ /day orally for 3 months leads to the reversal of dysplastic changes in leukoplakia ${ }^{12}$ and when given in the dosage of $16 \mathrm{mg} /$ day leads to substantials increase in the mouth opening in oral submucous fibrosis. ${ }^{13}$ The major dietary source of lycopene is tomatoes, with the lycopene in cooked tomatoes, tomato juice and tomato sauce included, being more bioavailable than that in raw tomatoes.

Flavonoids-They are polyphenolic compounds which are present in most plants. The main natural sources of flavonoids include green tea, grapes (red wine), apple, cocoa (chocolate), ginkgo biloba, soybean, curcuma, berries, onion, broccoli. They have been reported to prevent or delay a number of chronic and degenerative ailments such as cancer, cardiovascular diseases, arthritis, aging, cataract, memory loss, stroke, Alzheimer's disease, inflammation, infection. Green tea is a rich source of flavonoids, especially flavonols (catechins) and quercetin. Catechin levels are 4-6 times greater in green tea than in black tea. Many health benefits of green tea reside in its antioxidant, anticarcinogenic, antihypercholesterolemic, antibacterial (dental caries), anti-inflammatory activities ${ }^{14}$

Omega-3 and omega-6 fatty acids- They are essential long-chain polyunsaturated fatty acids. Dietary sources of omega- 6 fatty acids (linoleic acid) include vegetable oils, nuts, cereals, eggs, poultry. It is important to maintain an appropriate balance of omega-3s and omega- $6 \mathrm{~s}$ in the diet, as these two substances work together to promote health $^{1}$

\section{Recent antioxidant}

Decades of research have proven that precise combinations of the antioxidants phloretin, tetracurcuminoid and ferulic acid, including formulations applied topically, can neutralize cell-damaging free radicals, particularly those caused by UV rays, nicotine, alcohol, and hydrogen peroxide. ${ }^{15}$ Recent research has further confirmed that certain antioxidants, including phloretin, silymarin, and hesperetin, significantly inhibit the inflammatory response associated with Actinobacillus actinomycetemcomitans, one of the pathogens that cause periodontal disease. ${ }^{16}$ Lutein; dark green vegetables such as kale, broccoli, kiwi, sprout and spinach . Lignan ; oatmeal ,barley ,rye. Herbs; turmeric (curcumin), grape seed or pine bark extracts They can also provide powerful antioxidant protection for the body ${ }^{17}$.

\section{Antioxidant-enzymes}

Superoxide dismutase, catalase, and glutathione peroxidase serve as primary line of defense in destroying free radicals

\section{Mechanism of action of antioxidants ${ }^{3}$}

Antioxidants neutralize free radicals by donating one of their electrons, which ends the electron stealing reaction. The antioxidant nutrient, however, does not become a free radical by donating an electron because they are stable in either form. Important antioxidants include the following:

1. Chain breaking or scavenging ones, such as Vitamin E(alpha tocopherol), Vitamin C (ascorbic acid), or Vitamin A (beta carotene)

2. Preventative antioxidants that function largely by sequestering transition metal ions and preventing Fenton reactions and are therefore largely proteins by nature (e.g., albumin, transferring, or lactoferrin)

\section{Therapeutic use of antioxidants for oral Lesions ${ }^{4}$}

The possible uses of antioxidants for oral mucosal lesions include the following:

1. Prevention of lesions in high-risk individuals with mucosa that clinically appears normal with no history of either premalignant or malignant lesion 
2. The treatment of premalignant oral lesions

3. In patients who have had either premalignant or malignant oral lesions that have been successfully treated, in order to prevent recurrence of the treated initial lesion or to prevent the development of a second or a separate primary.

\begin{tabular}{|c|c|}
\hline \multirow{2}{*}{\multicolumn{2}{|c|}{$\begin{array}{c}\text { Protective Roles of Micronutrients in Carcinogenesis } \\
\text { Nutrient }\end{array}$}} \\
\hline & \\
\hline Beta- Carotene & $\begin{array}{l}\text { Precursor of vitamin A; anti-oxidant and free radical scavenging; } \\
\text { immunomodulation,stimulation of increase in numbers of T-helper and } \\
\text { NK cells as well as cells with IL-2receptors; inhibition of mutagenesis; } \\
\text { inhibition of cancer cell growth. }\end{array}$ \\
\hline $\begin{array}{l}\text { Vitamin A } \\
\text { (Retinoids) }\end{array}$ & $\begin{array}{l}\text { Inhibit keratinization and terminal dif--ferentiation of epidermal cells; } \\
\text { enhancement of cellular immunity; arrest/reverse leukoplakia } \\
\text { progression; induction of cytotoxic and cytostatic effects on cancer cells; } \\
\text { influence DNA, RNA, gene expression; interfere with carcinogenic } \\
\text { stimulation/ binding. }\end{array}$ \\
\hline $\begin{array}{l}\text { Vitamin E } \\
\text { (A-Tocopherol) }\end{array}$ & $\begin{array}{l}\text { Free radical scavenging; maintenance of membrane integrity, immune } \\
\text { function;inhibition of cancer cell growth/differentiation; cytotoxicity; } \\
\text { inhibits mutagenicity and nitrosamine formation; inhibition of } \\
\text { DNA,RNA, protein synthesis in cancer cells }\end{array}$ \\
\hline $\begin{array}{l}\text { Vitamin C } \\
\text { (Ascorbic Acid) }\end{array}$ & $\begin{array}{l}\text { Anti-oxidant; reduce vitamin E degradation;enhance chemotaxis, } \\
\text { phagocytosis, collagen synthesis; inhibit nitrosamine formation;enhance } \\
\text { detoxification via cytochrome P450; block formation of fecal mutagens; } \\
\text { reduce oncogene expression }{ }^{19}\end{array}$ \\
\hline
\end{tabular}

\section{Controversial areas in antioxidants therapy}

Current literature reports that a half century of data demonstrates the lack of predictability of antioxidant therapy and it has not been validated by the scientific method. Widespread use of antioxidants has failed to quell the current pandemic of cancer, diabetes, and cardiovascular disease or to stop or reverse the aging process. Antioxidant therapy in human reproductive medicine is controversial. High doses of Vitamin A may have embryo toxic and teratogenic effects. ${ }^{19}$ Large doses of ascorbic acid may be associated with the inhibition of ovarian steroidogenesis and increased probability of abortion. ${ }^{20}$

\section{Conclusion}

The antioxidant micronutrients are important not only for limiting oxidative and tissue damage, but also in preventing increased cytokine production, which is a result of prolonged activation of immune response. Dietary and other enzymatic antioxidants protect the lipids of lipoprotein and other biomembranes against oxidative damage by intercepting oxidants before they can attack the tissues. It is important to have an adequate antioxidant intake from both diet and supplementation if needed, and can be a valuable adjunct in the treatment of chronic inflammatory dental disorders. Antioxidants are being widely used in routine general clinical practice. They are also used in the prevention of cellular damage, the most common pathway for cancer, aging and a variety of disease. Dietary antioxidants like Vitamin C, Vitamin E, Carotenoids may also be of significance in the prevention of degenerative disease and maintenance of good health. There is now convincing evidence that foods containing antioxidants may be of major importance in disease prevention. .

\section{Free yourself from free radicals Eat more fruits and vegetables}

\section{References:}

[1]. Lien Ai Pham-Huy1 Free Radicals, Antioxidants in Disease and Health, www.ijbs.org Int J Biomed Sci vol. 4 no. 2 June 2008

[2]. Valko M, Rhodes CJ, Moncol J, Izakovic M, et al. Free radicals, metals and antioxidants in oxidative stress-induced cancer. Minireview. Chem Biol Interact. 2006;160: 1-40.

[3]. Arivind Shetty et al Journal of Indian Academy of Oral Medicine and Radiology / Jan-Mar 2009 / Volume 21 / Issue 1

[4]. Neelu Shetti Antioxidants: its beneficial role against health damaging free.Radical World Journal of Science and Technology 2011, 1(11): 46-51 ISSN: 2231 - 2587 www. worldjournalofscience.com

[5]. Sumit Bhateja, IJPSR, 2012; Vol. 3(7): 1971-1975 ISSN: 0975

[6]. Iain L, C . Chapple, John B Mathews., (2000). The role of reactive oxygen and antioxidants species in periodontal destruction .Periodontology, $43: 160-232$

[7]. Li Y, Schellhorn HE. New developments and novel therapeutic perspectives for vitamin C. Critical Review. J Nutr 2007;137:217184. 
[8]. Mayo Clinic Medical Information. Drugs and supplements. Vitamin E. 2005 http://www.mayoclinic.com/health/vitamine/NS_patient vitamin-e. .

[9]. Miller ER, Pastor-Barriuso R, Dalal D, et al. Meta-analysis: high-dosage Vitamin E supplementation may increase all-cause mortality, Ann Intern Med 2005;142:37-46.

[10]. Willcox JK, Ash SL, Catignani GL. Antioxidants and prevention of chronic disease. Review. Crit Rev Food Sci Nutr 2004;44:275-95

[11]. Pham-Huy C., Nguyen P., Marchand V., et al. Selenium and tobacco smoke tars: In vitro effects on different immunocompetent cells. Toxicology 2001;164:111-2. Presented in International Congress of Toxicology XI, Brisbane (Australia), 7-12 July 2001.)

[12]. Rao V. Bioavailability and in vivo antioxidant properties of lycopene from tomato products and their possible role in the prevention of cancer. Nutr Cancer 1998;31:199-203.),[6]

[13]. World Journal of Science and Technology | www.worldjournalofscience.com | 2011 | 1(11): 46-51

[14]. Pham-Huy NLA, He H and Pham-Huy C. Green tea and health. An overview. J Food Agric Environ (JFAE) 2008;6:6-13.).

[15]. San Miguel S., et al. Bioactive antioxidant mixtures promote proliferation and migration on human oral fibroblasts. Archives of Oral Biology 2011;doi:10.1015/jarchoralbil.2011.01.001.

[16]. Nares S. The anti-oxidants phloretin, silymarin, hesperetin and resveratrol inhibit the inflammatory response of human blood mononuclear cells to lipopolysaccharide.

[17]. G. h. naik, Res. Chem. Intermed., Vol. 31, No. 1-3, pp. 145-151 (2005) Evaluating the antioxidant activity of different plant extracts and herbal formulations

[18]. Enwonwu, Meeks., (1995). Bionutrition and Oral cancer in humans . Crit Rev Oral Biol Med, 6(1) : 5 -17.

[19]. Carnelio S, Khan SA. Free radicals and antioxidant therapy in clinical practice: To be or not to be. J Coll Physicians Surg Pak 2007;17:173-4.

[20]. Meyers DG, Maloley PA, Weeks D. Safety of antioxidant vitamins. Arch Intern Med 1996;156:925-35. 